\title{
Impact of a service learning experience on the intercultural sensitivity of nursing students
}

\author{
Catherine S. Thomas, Leona Konieczny* \\ Department of Nursing, Central Connecticut State University, United States
}

Received: February 22, 2017

Accepted: March 27, 2017

Online Published: March 30, 2017

DOI: $10.5430 /$ cns.v5n2p41

URL: https://doi.org/10.5430/cns.v5n2p41

\begin{abstract}
Service learning (SL) is an educational strategy to develop intercultural sensitivity. Nursing students benefit from the opportunity to care for persons from diverse cultures. Social awareness and making significant social contributions is part of professional nursing. Nursing students are educated to provide culturally sensitive, person-centered care incorporating the values of social justice, diversity, and global awareness. The SL experience was independent from a specific nursing course and exclusive from a designated clinical practicum. This descriptive quantitative study examines the impact of a SL experience in Appalachia on intercultural sensitivity. Intercultural sensitivity is in the affective domain of intercultural communication. Appalachia has distinct cultural differenences related to both geographical and socioeconomic factors. Intercultural sensitivity is in the affective dimension of intercultural communication competence. The Intercultural Sensitivity Scale (ISS), developed by Chen \& Starosta, was the instrument used for measurement. Study findings demonstrate an impact on interaction engagement and interaction attentiveness for study participants.
\end{abstract}

Key Words: Service learning, Nursing students, Intercultural communication, Affective domain

\section{INTRODUCTION}

\subsection{Developing cultural sensitivity in nursing students}

Achieving cultural competence is a progressive journey for nurses, which begins during their time as nursing students. The need for nurses who are culturally aware, engaged and sensitive to the diverse needs of the populations that nurses care for has long been established by external and internal stakeholders of the health care system. In response to that need, implementation of various teaching methods have focused on developing a foundation of cultural competence widely included in nursing curricula. One teaching method that frequently incorporates cultural exposure, is the learning pedagogy of service learning (SL). SL has become increasingly commonplace in colleges and universities in the United
States (US). Multiple studies have suggested that there are a variety of benefits that result from SL. ${ }^{[1]}$

\subsection{Cultural differences in Appalachia}

For this study, the volunteer experience was a two day mobile medical clinic which offered free medical, vision and dental care. Located in rural eastern Tennessee, the area is designated as part of the Appalachian region by the Appalachian Regional Commission. ${ }^{[2]}$ The Appalachian region has marked differences than the remainder of the US; it is more rural, and despite moderate gains from various public and private interventions, the poverty rate for many counties in Appalachia continues to be significant. Appalachian people should be considered as having a distinct culture,

*Correspondence: Leona Konieczny; Email: konieczny@ @csu.edu; Address: Department of Nursing, Central Connecticut State University, New Britain, CT, 06050, United States. 
comprised of several ancestries: Native Americans, Irish, English, Scotch, German and Polish, that have integrated across the region. The geography has contributed to the uniqueness of Appalachia, the mountains keeping it relatively isolated from the remainder of the US and from outside influences. Appalachians expect others to respect their freedom and independence, being self-sufficient is valued. Appalachian people frequently are resistant to change, slow to accept outsiders and reluctant to accept authority. ${ }^{[2,3]}$

Varied definitions of SL exist. For this study, it is defined as a pedagogical approach that is experiential in nature, which intentionally supports the achievement of institution and program goals through structured reciprocal learning activities. ${ }^{[4,5]}$ Those activities provide students the opportunity to experience civic engagement and cultural diversity. With the incorporation of reflection, students are allowed the opportunity of a guided exploration of self-awareness and preconceptions that they may have had prior to the completion of SL activities. ${ }^{[4,5]}$

\subsection{Literature review of SL}

The interest in the benefits of SL for students in other health disciplines is evident by recent articles in the literature, discussing the value of SL for public health students and student perceptions of their needs related to preparation for their SL experiences. ${ }^{[6,7]}$ While the research on SL in other disciplines than nursing has demonstrated benefits that extend from increased grade point averages to civic responsibility, along with life skills, the scope of those benefits has not been empirically measured in a systematic way. ${ }^{[1]}$

A review of literature on SL in nursing education concluded more research was necessary, and proposed that there is much promise in relation to stimulating social change, allowing students to embrace the role of change agent, a chance to experience diversity in alternate settings, as well as to develop into active engaged citizens. ${ }^{[8]}$ The review recommended debriefing as SL can be emotional for students. This may be related to life experience and development variations among students.

The research on SL in nursing education has expanded since that literature review. Three quantitative studies on the effect of SL completed with varied approaches on multiple variables found improvements in civic engagement scores, attitudes towards homelessness, leadership skills and social justice interests for nursing students. ${ }^{[9-11]}$ One qualitative study found that the students perceived the experience as challenging their understanding of self, and contributing to their becoming a nurse, and learning to care. ${ }^{[5]}$ The SL experiences in the above mentioned studies took place as part of a specific course. One mixed methods study described SL that impacted cultural self-efficacy, confidence, and awareness that was a non-course based experience. ${ }^{[12]}$

\subsection{Service to culturally distinct persons and the impact on intercultural sensitivity}

The hypothesis is that providing service to culturally distinct persons may have on impact on intercultural sensitivity. Intercultural sensitivity is in the affective domain of intercultural communication. The pilot study examines if there are differences in intercultural sensitivity as a result of SL.

\section{METHOD}

The study received approval from the Human Studies Council at the university. Intercultural Sensitivity, the affective dimension of intercultural communication competence, was measured by the Intercultural Sensitivity Scale (ISS). This instrument, new to nursing research, was selected on its unique ability to measure multiple aspects of sensitivity towards others. The ISS developed by Chen and Starosta, is a 24 item instrument, scored on a Likert-type scale ranging from 1 (strongly disagree) to 5 (strongly agree) to measure a total of five components of intercultural sensitivity: interaction engagement ( 7 items), respect for cultural difference (6 items), interaction confidence ( 5 items), interaction enjoyment (3 items), and interaction attentiveness (3 items). ${ }^{[13]}$ The instrument was used with permission of the author. The reliability coefficient for this instrument is $0.86-0.88$. All study participates received verbal and written description of the study.

\subsection{Design}

Two days prior to departure, written consent was obtained from the participants. Prior to SL, the participants completed the demographics characteristics sheet and ISS. Then upon return from SL, the ISS was completed within one week.

\subsection{Setting}

The baccalaureate nursing program is part of a public state regional university located in the North East section of the US. One of the four distinctive elements of the mission for the university is community engagement with the goal of promoting higher level learning and cultural understanding for students and faculty. The nursing department mission is congruent in that the expectation is for the students to develop social awareness and make socially significant contributions to society. The SL experience was located in a small town in Monroe County, Tennessee, which is part of the Appalachian region.

Monroe County in Tennessee has a poverty level of $20.6 \%$, with $17.6 \%$ of the population under the age of 65 who do 
not have health insurance. In comparison, the county that the university is located in and where the majority of clinical experiences occur, has a poverty rate of $12.2 \%$ for individuals and for children (under 18) of $17.9 \%$, with $10.3 \%$ of the population under the age of 65 who do not have health insurance. ${ }^{[14]}$

\subsection{Sampling procedures}

The study sample was a convenience sample of the students participating in the SL. The students applied for SL. An essay describing personal reasons for participation in the experience was done by students. The Department of Nursing faculty members were blinded to the applicants' names. As this was a pilot study, the sample size was limited to ten students due to the cap on number of nursing students imposed by the volunteer organization operating the mobile healthcare clinics and due to financial costs for travel and lodging.

\subsubsection{Demographic variables of study sample}

Participants were asked to identify the demographic variables of age, gender, whether they have lived in another state, experience working in healthcare setting (excluding assigned clinical experiences), experience volunteering in community setting, experience traveling outside of the state, or experience traveling outside of the US.

\subsubsection{Research design}

Study sample participants anonymously completed the demographic information and the ISS. The data collected were the pre SL and post SL ISS. The service provided to culturally distinct persons was determined by the mobile volunteer organization that operated the healthcare clinics. The volunteer organization requires that the ratio of nursing faculty to nursing students is 1:5. There was compliance to this requirement. There was not a control group. The collection of data was done within subjects. Nine students elected to participate in data collection.

\section{RESUlts}

The ISS instrument was scored using the instruction provided by the author of the instrument. Data were used from participants that completed all items on the instrument both before and after the SL experience. Data analysis was performed using the Statistical Package for the Social Sciences (SPSS) version 22.0.

All nine participants were female, the age range was 21 37 , the mean age was 23.67 , the median was 22 , and the mode was 21 . Two of the participants had lived out of state (22.2\%), the remaining 7 had not lived out of state $(77.8 \%)$. Four participants had healthcare work experience $(44.4 \%)$. Eight of the participants had previous experience volunteering $(88.9 \%)$. All of the participants had traveled outside of the state, and $7(77.8 \%)$ had traveled outside of the US. For demographic data analysis, the yes responses were coded as 1 , and the no responses were coded as 2 .

For all participants, the pre and post item analysis was completed by conducting paired sample $t$-tests were run on statements pre and post mission experience, there were 3 statements that were significant at 0.10 . Because this was a pilot study with a low $n$, the decision was made to accept a significance level of 0.10 .

Under the component of interaction engagement, there were two statements that were significant as a result of SL. Statement 11 , which measured a waiting approach when forming an impression of culturally distinct counterparts was significant at 0.081. Statement 23, which measured their demonstration of understanding through verbal or non-verbal cues was significant at 0.051 . Under the component of interaction attentiveness, there was one statement that was significant. Statement 14, which measured their attentiveness when interacting with others was significant at 0.081 (see Table 1).

Table 1. ISS pre and post SL paired samples $t$-test

\begin{tabular}{|c|c|}
\hline ISS statement & $p$-value \\
\hline 11. I tend to wait before forming an impression of culturally-distinct counterparts. (Interaction Engagement) & .081 \\
\hline 14. I am very observant when interacting with people from different cultures. (Interaction Attentiveness) & .081 \\
\hline 23. I often show my culturally-distinct counterpart my understanding through verbal or nonverbal cues. (Interaction Engagement) & .051 \\
\hline
\end{tabular}

In examining the demographic variables with pre and post items, there were three significant differences. For healthcare work experience and statement 23 , I often show my culturally-distinct counterpart my understanding through verbal or nonverbal cues, pre SL demonstrated significance at 0.011. For lived other than CT and statement 23, I often show my culturally-distinct counterpart my understanding through verbal or nonverbal cues, pre SL demonstrated significance at 0.059 , and post SL at 0.034. For travel outside of US and statement 14 , I am very observant when interacting with people from different cultures, pre SL demonstrated 0.052, and post SL 0.034, and pre SL statement 23 was 0.094 . 


\section{Discussion}

It may appear that a significant difference in three statements is a mild response. Rather because the concepts of cultural competence and therapeutic communication are taught in the first nursing courses which start in the fall semester of the sophomore year, the results are important. So even though the study participants were concluding the end of the spring semester of the junior year, intercultural sensitivity was affected by the SL experience. The SL experience affected the participants waiting to form an impression of culturally distinct counterparts. This is important because the experience strengthened the non-judgmental approach in communicating with others. Working with others from a different culture reinforced the importance of not rushing to make judgments. In communication, it may take time to accurately understand the other person. The experience also reinforced the participants' demonstration of understanding through verbal or nonverbal cues. This is another behavior that supports effective communication where the nurse needs to communicate effectively in words and body language to engage with others. The participants' self-rating demonstrated growth in engaging in communication to connect with others from a different culture. Another area positively affected was in being observant when interacting with people from different cultures. The first part of the physical assessment process is inspection. The SL experience highlighted the importance of observation during communication. The participants benefited from gathering data about the person, appearance, clothing, and other visual information. The SL experience bolstered the importance of observing while communicating. This is important for nursing students who need this data to make assessments, plan interventions, and evaluate efficacy of care.

A strength of this pilot study is that it is not connected to a specific course within the nursing curriculum. Students, who may have financial or time limitations presented by international travel, were eligible to participate. Other literature as described in the literature review examine local SL or international SL. This pilot study differs in that it was a national experience to a culturally distinct region within the US. The ISS has been used in other disciplines with communication and business administration students. ${ }^{[15,16]}$ This pilot study is the first to utilize the ISS instrument with nursing students.

The information from this pilot study will serve to change and improve the implementation of similar SL experiences in the future. Although it will not affect student selection, knowledge about the students' work experience and travel may positively affect orientation for future opportunities for service. Students who had healthcare work experience were more perceptive about demonstrating understanding verbally and nonverbally. This will change the orientation process moving forward to prepare students without healthcare work experience to facilitate communication with persons from a different culture. Students might benefit from role play exercise or simulation to gain awareness about spoken communication and body language prior to travel. Study participants who had lived outside the state may be used as peer supports before and during the experience to model for the students, who had only resided in one state, effective ways to communicate understanding. This knowledge may affect groupings of students to include a peer support in each group. Faculty who are supervising these students can build upon the experiential learning that occurs when travelling outside the US. Drawing analogies between a prior travel experience and the culturally distinct SL experience, may decrease anxiety about communicating with others from an unfamiliar location. Part of the orientation or debriefing may be a matching exercise where students are presented with a scenario and then describe how a communication strategy could be applied in multiple situations or environments. This may decrease unease among students who may feel the experience in a different culture is totally new and potentially overwhelming. This same exercise may be applied during a clinical experience when the student is caring for someone from a different culture.

This pilot study has resulted in lessons learned by the faculty. There are some study limitations. First, because, it was the initial experience, only ten students participated which resulted in a small sample. Second, the mobile medical clinic was only moderate in size. There is potential that the results would vary if the clinic were larger thus serving more persons. Third, the two groups of participants had different amounts of time communicating with persons from the Appalachian area. One group of five students had more direct contact than the other due to the capacity of providers at the clinic. This may have impacted the findings of the study. A fourth consideration is that study participants did have a short orientation about the SL prior to completing the first ISS. Lastly, on the day of departure the group visited the National Center for Civil and Human Rights in Atlanta prior to the plane flight home. While this may have affected participants' responses, the quality of the exhibits at the museum outweighed the consideration.

It is the expectation of the faculty to build upon the findings in this descriptive study to plan and implement further SL. The results from this pilot study are being used to guide the design of a study for the next SL experience. The future study will include a control group as well as the use of an additional instrument measuring civic engagement. Pre and post SL data collection will occur at the beginning and end of the academic semester. Additionally the length of the 
SL will be increased to provide nursing students with more interaction opportunities. Further areas to be studied include intercultural experiences in varying culturally distinct regions within the US and taking participants from different levels within the program. This experience met the mission of the university and the goal of the department of nursing.

CONFLiCTS OF InTEREST Disclosure

The authors declare they have no conflict of interest.

\section{REFERENCES}

[1] Toncar MF, Reid JS, Burns DJ, et al. Uniform assessment of the benefits of service learning: the development, evaluation, and implementation of the SELEB scale. Journal of Marketing Theory and Practice. 2006; 14: 223-238. https://doi.org/10.2753/MTP1 069-6679140304

[2] Appalachian Regional Commission [Internet]. Office of the Inspector General. [cited 2017 Jan 12]. Available from: http: //www . arc .go v/index.asp

[3] Appalachian Regional Ministry [Internet]. North American Mission Board; no date [cited 2017 Mar 2]. Available from: http: //www.namb.net

[4] Stallwood LG, Groh CJ. Service-learning in the nursing curriculum: Are we at the level of evidence-based practice? Nurs Educ Perspect. 2011 September/October; 32(5): 297-301. PMid: 22029240. https://doi.org/10.5480/1536-5026-32.5.297

[5] Schofield R, Allan M, Jewiss T, et al. Knowing self and caring through service learning. Int J of Nurs Educ Scholarsh. 2013; 10(1): 267-274. PMid: 24317787. https://doi.org/10.1515/ijne s-2013-0009

[6] Sabo S, De Zapien J, Teufel-Shone N, et al. Service learning: A vehicle for building health equity and eliminating health disparities. Am J Public Health. 2015. Supplement 1; 105(S1): S38-S43.

[7] Wallace JP, Blinkhorn FA, Blinkhorn AS. Dental hygiene students' views on a service-learning residential aged care placement program. J Dent Hyg. 2014; 88(5): 309-315. PMid: 25325727.

[8] Gillis A, Mac Lellan M. Service learning with vulnerable populations: Review of literature. J Nurs Scholarsh. 2010; 7(1): 1-27. https://doi.org/10.2202/1548-923x. 2041
[9] Groh CJ, Stallwood LG, Daniels JJ. Service learning in nursing education: Its impact on leadership and social justice. Nurs Educ Perspect. 2011; 2(6): 400-405. https : //doi .org/10.5480/1536 $-5026-32.6 .400$

[10] Loewenson KM, Hunt RJ. Transforming attitudes of nursing students: Evaluating a service-learning experience. J Nurs Educ. 2011; 50(6): 345-349. PMid: 21524020. https : //doi .org/10.3928/014848 34-20110415-03

[11] Nokes KM, Nickitas DM, Keida R, et al. Does service learning increase cultural competency, critical thinking, and civic engagement? J Nurs Educ. 2005; 44(2): 65-70. PMid: 15719713.

[12] Long T. Influence of international service learning on nursing students' self-efficacy towards cultural competence. J Cult Divers. 2016; 23(1): 8-33.

[13] Chen GM, Starosta WJ. The Development and Validation of the Intercultural Sensitivity Scale. Human Communication. 2000; 3: 1-15.

[14] United States Census Bureau [Internet]. US Department of Commerce; no date [cited 2017 Jan 12]. Available from: http://www . census.gov

[15] Portalla T, Chen GM. The Development and Validation of the Intercultural Effectiveness Scale. Intercultural Communication Studies. 2010; 19(3): 21-27.

[16] Fritz W, Mollenberg, A, Chen GM. Measuring Intercultural Sensitivity in Different Cultural Context. Paper presented at: International Association for Intercultural Communication Studies Meeting; 2001; Hong Kong, CHN. 